\title{
CYP2C9 Mutation Affecting the Individual Variability of Warfarin Dose Requirement
}

\author{
Young Bum Kim, M.D., Moon Ju Ko, M.D. , Dae Gu Lee, M.D. ${ }^{2}$, Jong Gul Do, M.D. ${ }^{2}$, Ji Hye Hwang, M.D. ${ }^{2}$ \\ Department of Rehabilitation Medicine, Pusan National University Yangsan Hospital, Yangsan 626-770, \\ ${ }^{1}$ Parkside Rehabilitation Hospital, Pusan 608-020, ${ }^{2}$ Departments of Physical and Rehabilitation Medicine, \\ Samsung Medical Center, Sungkyunkwan University School of Medicine, Seoul 135-710, Korea
}

Warfarin is a frequently prescribed anticoagulant in rehabilitation patients. Adverse drug reactions of warfarin were reported as bleeding and cutaneous microvascular thrombosis. Major bleeding, such as intracranial hemorrhage and psoas hematoma, in patients receiving anticoagulation therapy is a rare condition, but sometimes very serious complication that can even be fatal. Patient-specific factors (eg, age, body size, race, concurrent diseases, and medications) explain some of the individual variability in warfarin dose, but genetic factors, which influence warfarin response, explain a significantly higher proportion of the variability in the dose. There are two identified genes that are responsible for the main proportion of the genetic effect: CYP2C9, which codes for the enzyme cytochrome P450 2C9 that metabolizes S-warfarin, and VKORC1, which codes for warfarin's target, vitamin $\mathrm{K}$ epoxide reductase. We report a case of intolerance to warfarin dosing, due to impaired drug metabolism in a patient with CYP2C $9 * 1 / * 3$ and VKORC $1173 T$ T. Fortunately, there are no severe complications.

Key Words Warfarin, CYP2C9, Vitamin K epoxidase

\section{INTRODUCTION}

Warfarin is one of the drugs that are most often used in rehabilitation medicine. As an important therapy to prevent the relapse of cerebral infarction and improve neurological damage, it is commonly used in the rehabilitation of subacute stroke and for patients with spinal

Received August 1, 2011; Accepted November 21, 2011

Corresponding author: Ji Hye Hwang

Departments of Physical and Rehabilitation Medicine, Samsung Medical Center, Sungkyunkwan University School of Medicine, 50, Irwon-dong, Gangnam-gu, Seoul 135-710, Korea

Tel: +82-2-3410-2818, Fax: +82-2-3410-0052, E-mail: hwanglee@skku.edu (C) This is an open-access article distributed under the terms of the Creative Commons Attribution Non-Commercial License (http:// creativecommons.org/licenses/by-nc/3.0) which permits unrestricted noncommercial use, distribution, and reproduction in any medium, provided the original work is properly cited.

Copyright $\odot 2012$ by Korean Academy of Rehabilitation Medicine cord injuries, as well as for the prevention and treatment of frequent deep vein thrombosis and pulmonary thromboemblolism. However, since warfarin has a narrow therapeutic range and a lot of differences in the sensitivity between individuals, it needs appropriate controlled doses, and since it has a high risk of hemorrhage, cautious administration is required and interactions with other drugs and foods, as well as pharmacokinetic phases by age should always be carefully observed. ${ }^{1}$

Differences in individuals' sensitivities to warfarin are known to be affected by other drugs, foods and genetic diversities. Of these, understanding the genetic diversities has been achieved through continuous genetic analyses, and for this, the U.S. Food and Drug Administration (FDA) currently recommends a genetic test before an anticoagulation therapy. ${ }^{2}$

Single Nucleotide Polymorphisms (SNPs) are known 
to affect the maintained dose of warfarin for an appropriate anticoagulation effect. Some SNPs are located in cytochrome P450 2C9 (CYP2C9), acting as a catalyst of the metabolism of warfarin, while others are located in Vitamin K epoxide Reductase Complex 1 (VKORC1), and warfarin restrains VKORC1, which restrains Vitamin K-dependent Clotting Factors. ${ }^{3,4}$ So, the genetic polymorphism of CYP2C9 and VKORC1 affects the diversities of the maintained dose of warfarin, not only of the individual patients, but also the nation.

There have been many researches on CYP2C9 mutation affecting the individual variability of warfarin dose requirement, but very few report in clinics area.

Severe hemorrhagic complications frequently occur during an acute rehabilitation treatment. Individual variability of warfarin dose requirement is one of the reasons that occur these complications, so It is thought that the knowledge of CYP2C9 mutation, affecting the individual variability of warfarin dose requirement, is actually very important for physiatrists.

The authors experienced a case of a noticeable prolongation of PT (INR) at the inducement time that determines the maintained dose of warfarin during anticoagulation treatment and the subsequent administration of extremely low doses maintained extremely low, found the allelic mutations of CYP2C9 and VKORC1, and report them with literature review.

\section{CASE REPORT}

A 77-year-old female patient with the main complaint of gait disturbance was transferred to the Department of Rehabilitation Medicine. The patient had been diagnosed with colorectal cancer 6 years ago and received right Hemicolectomy and Total Hip Replacement Arthroplasty due to the metastasis of cancer to her right hip joint, two days before. In an ultrasonography on her leg veins, as an examination carried out two days before the surgery, a blood clot on the posterior tibial vein and thromboembolism on the right upper lobe, in addition to mesenchyma on the left inferior lobe from a chest CT were observed. As such, an anticoagulation treatment of thrombotic lesion was started on the next day from the surgery. The patient, on the day of the transfer to the Department of Rehabilitation Medicine, was taking oxcodone $(20 \mathrm{mg} /$ day), atorvastatin $(20 \mathrm{mg} /$ day), gabapentin $(900 \mathrm{mg} /$ day), levosulpiride (25 mg/day), and cetamadol (R) (325 $\mathrm{mg} / 37.5 \mathrm{mg}$ ), three times a day. Her weight was $57.6 \mathrm{~kg}$ and her height was $160 \mathrm{~cm}$. There was no special opinion on the basic blood chemistry and electrolyte, and on the first blood coagulation test, PT was 14.2 seconds and INR was 1.10 , which were normal.

As an anticoagulation treatment, the administration of warfarin started using enoxaparin $(60 \mathrm{mg} /$ day). The initial dose of warfarin was $8 \mathrm{mg}$ after dinner on the first day, and PT next morning was 15.8 seconds and INR was measured 1.26. In the evening on the same day, $8 \mathrm{mg}$ was administered again, and PT on the following morning was 62.6 seconds and INR was 7.33. In addition, the value of the coagulation remarkably increased. Thus, the authors, first considered the possibility of overdose of warfarin and an interaction with other drugs. The administration of cetamadol and warfarin was stopped, and Vitamin K $10 \mathrm{mg}$ IV was administered to prevent copious bleeding, and then stopped the rehabilitation and radiation treatments. On the following morning, PT was reduced to 23.0 seconds and INR to 2.03, so warfarin was administered again for two days ( $2 \mathrm{mg} /$ day), and the next morning's PT remarkably increased to 69.1 seconds and INR to 8.31. Again, Vitamin K $10 \mathrm{mg}$ IV was administered, and the administration of warfarin was stopped. Four days after, INR was maintained at more than 4.0 and on the fifth day, PT was reduced to 20.8 and INR to 1.79 . As a result, it turned out to be CYP2C $9 * 1 / * 3$ genotype, in which the heterozygous A1075C (Ile359Leu) polymorphism was found on the result of a CYP2C9 genotype analysis, in which a Polymerase Chain Reaction (PCR) and Direct Sequencing method were carried out, and an intermediate metabolizer with deteriorated activation of enzyme compared to that of a wild type person. It was found that VKORC1 genotype as 1173TT (homozygous) had a genetically high warfarin sensitivity. Thus, the authors started to find the maintained dose of warfarin of about $0.5-1 \mathrm{mg} /$ day again, and with the dose of $0.5 \mathrm{mg} /$ day, for the patient's value of coagulation, PT and INR was maintained at $21 \mathrm{sec}-$ onds and about 1.9, respectively, and two weeks later, the radiation treatment was terminated. Consequently, the patient was discharged from the hospital with the proper function of independent walking, using a rolling walker.

\section{DISCUSSION}

The half period of warfarin in blood is 36 hours, on average, and $99 \%$ of the drugs that are absorbed into blood 
vessels are combined with albumin in the blood and disturbs the hepatic synthesis of Vitamin K-dependent coagulation factors, to induce anticoagulation. It is an effective oral anticoagulant for the prevention and treatment of thromboembolism, but attention should be paid in the use of it since it has a limited therapeutic range, a high risk of hemorrhage and interaction with other drugs and food. ${ }^{1}$

Cruickshank et al. ${ }^{5}$ reported that severe hemorrhage, at about $1.0-3.0 \%$ and light hemorrhage at about $4.8-9.5 \%$, occur in warfarin-dosed patients each year. Landelfeld and Beyth reported that the risk of hemorrhage in the group of patients who received warfarin treatment was $0.6-9.6 \%$, which is five times higher than that in a group of those without warfarin administration. ${ }^{6}$

Individuals' sensitivities to warfarin differ, depending on the various factors, such as age, gender, weight, genetic mutation and drugs used, and of these, CYP2C9 and VKORC1 genotype is an important genetic element that can explain the differences in warfarin capacity between individuals. The metabolism of warfarin is stimulated by CYP2C9 enzyme, which metabolizes S-warfarin to inert S-7-hydroxywarfarin. Patients with genetically mutated CYP2C9 become very sensitive to warfarin, so when the standard dose was used, they show over-anticoagulation. There are more than 20 alleles of CYP2CP mutation reported. Of these, CYP2C ${ }^{*} 2$ and CYP2C $9 * 3$ are common alleles that reduce the warfarin metabolism; CYP2C9*3 has been reported $6-10 \%$ in Caucasians and $2-5 \%$ in Asians, and in a study on Koreans, CYP2C9*1 was reported in $93 \%$, CYP2C9*3 in $6 \%$ and CYP2C9* 13 in $0.6 \%$, while CYP2C $9 * 2,{ }^{*} 4,{ }^{*} 5,{ }^{*} 11$ was not found. Also, CYP2C $9 * 1 /{ }^{*} 1$, ${ }^{*} 1 /{ }^{*} 3$ and ${ }^{*} 1 /{ }^{*} 13$ genotypes were reported to be found in $86.9 \%, 12 \%$ and $1.1 \%$, respectively. ${ }^{7,8}$ In addition, warfarin shows an anticoagulation by restraining VKORC1, which mediates Vitamin K activation, reducing 2,3-epoxide, and VKORC1 has 1173TT, CT and CC types. In addition, the frequency of these polymorphisms also differs depending on the nations: It has been reported that $80 \%$ of Japanese are TT type; and $27 \%$ of Israeli are TT type. ${ }^{9}$ Also, in Koreans, 1173TT type is most common and it has been reported that $1173 \mathrm{C}$ alleles are found in $9.7 \%$ of them, and for VKORC1 1173CT type, the maintained dose of warfarin at $5.5 \mathrm{mg} /$ day is used on average, while for 1173TT type, the maintained dose at $3.8 \mathrm{mg} /$ day is used, so it has been reported that 1173TT type exhibits a higher sensitivity to warfarin than 1173CT type does. ${ }^{10}$
For most patients who need anticoagulation treatment, usually the maintained dose of warfarin is $4-6 \mathrm{mg} /$ day; however, in this case, the intended PT (INR) values could be maintained at $0.5 \mathrm{mg} /$ day, which are very low warfarin doses. Although there was a very high risk of serious complications, like copious hemorrhage and etc., fortunately, no special complications occurred.

The polymorphisms of CYP2C9 and VKORC1 genes affect the pharmacokinetics of warfarin. Therefore, for a safer and a more effective warfarin medication, it is desirable to consider the genotypes of individual patients. However, in the situations of medical treatments in Korea, if the costs are taken into consideration, it is difficult to test CYP2C9 and VKORC1 genes for all patients who need warfarin medication. Thus, administrating warfarin, if there is any abnormal metabolism, with an especially close PT (INR) progress observation, DNA tests should be carried out, keeping the possibilities by CYP2C9 and VKORC1 genes in mind.

\section{REFERENCES}

1. Hirsh J, Dalen J, Anderson DR, Poller L, Bussey H, Ansell J, Deykin D. Oral anticoagulants: mechanism of action, clinical effectiveness, and optimal therapeutic range. Chest 2001; 119 Suppl 1: 8S-21S

2. Schwarz UI, Ritchie MD, Bradford Y, Li C, Dudek SM, Frye-Anderson A, Kim RB, Roden DM, Stein CM. Genetic determinants of response to warfarin during initial anticoagulation. N Engl J Med 2008; 358: 999-1008

3. Higashi MK, Veenstra DL, Kondo LM, Wittkowsky AK, Srinouanprachanh SL, Farin FM, Rettie AE. Association between CYP2C9 genetic variants and anticoagulation-related outcomes during warfarin therapy. JAMA 2002; 287: 1690-1698

4. Aithal GP, Day CP, Kesteven PJ, Daly AK. Association of polymorphisms in the cytochrome P450 CYP2C9 with warfarin dose requirement and risk of bleeding complications. Lancet 1999; 353: 717-719

5. Cruickshank J, Ragg M, Eddey D. Warfarin toxicity in the emergency department: recommendations for management. Emerg Med (Fremantle) 2001; 13: 91-97

6. Moyer TP, O'Kane DJ, Baudhuin LM, Wiley CL, Fortini A, Fisher PK, Dupras DM, Chaudhry R, Thapa P, Zinsmeister AR, et al. Warfarin sensitivity genotyping: a review of the literature and summary of patient experience. Mayo Clin Proc 2009; 84: 1079-1094 
7. Takahashi H, Echizen H. Pharmacogenetics of CYP2C9 and interindividual variability in anticoagulant response to warfarin. Pharmacogenomics J 2003; 3: 202-214

8. Bae JW, Kim HK, Kim JH, Yang SI, Kim MJ, Jang CG, Park YS, Lee SY. Allele and genotype frequencies of CYP2C9 in a Korean population. Br J Clin Pharmacol 2005; 60: 418-422

9. Nakai K, Tsuboi J, Okabayashi H, Fukuhiro Y, Oka T, Habano W, Fukushima N, Nakai K, Obara W, Fujioka
T, et al. Ethnic differences in the VKORC1 gene polymorphism and an association with warfarin dosage requirements in cardiovascular surgery patients. Pharmacogenomics 2007; 8: 713-719

10. Cho HJ, Sohn KH, Park HM, Lee KH, Choi B, Kim S, Kim JS, On YK, Chun MR, Kim HJ, et al. Factors affecting the interindividual variability of warfarin dose requirement in adult Korean patients. Pharmacogenomics 2007; 8: 329-337 